\title{
Barriers to sharing information with schools
}

Summarised by Dr. Jessica K Edwards

A recent study by Tania Hart and Michelle O'Reilly has found that the exchange of information between Child and Adolescent Mental Health Services (CAMHS) and schools needs improving to sufficiently support the educational needs of young people with emotional mental health difficulties. The study explored sensitive information exchange from the perspective of young CAMHS users, whilst incorporating the views of their parents and teachers. Key concerns raised by the participants included:

(1) that many teachers have insufficient understanding of mental health issues, which impacts on confidence about confidential information handling; (2) that a collaborative decision-making process between CAMHS and families should be established, to determine what is the key information that should be shared with school; (3) that CAMHS professionals should share information with schools as appropriate; and (4) that the child's wishes with regards to information sharing should be included in the decision making. The majority of affected participants felt their recovery would be facilitated if teaching staff had a greater understanding of mental health difficulties. The researchers conclude that secure procedures to navigate the communication barriers between families, CAMHS and schools are urgently required, and that such protocols and policy should consider the voice of the affected child.

Hart, T. \&O'Reilly, M. (2017), "The challenges of sharing information when a young person is experiencing severe emotional difficulties": implications for schools and CAMHS. doi:10.1111/camh.12245 